\title{
Intracellular inflammatory and antioxidant pathways in postmortem frontal cortex of subjects with major depression: effect of antidepressants
}

David Martín-Hernández ${ }^{1,2,3+}$, Javier R. Caso ${ }^{1,2,3^{*}+}$ (D) J. Javier Meana ${ }^{1,4,5}$, Luis F. Callado ${ }^{1,4,5}$, José L. M. Madrigal ${ }^{1,2,3}$, Borja García-Bueno ${ }^{1,2,3}$ and Juan C. Leza ${ }^{1,2,3}$

\begin{abstract}
Background: Studies show that Toll-like receptors (TLRs), members of the innate immune system, might participate in the pathogenesis of the major depressive disorder (MDD). However, evidence of this participation in the brain of patients with MDD has been elusive.

Methods: This work explores whether the protein expression by immunodetection assays (Western blot) of elements of TLR-4 pathways controlling inflammation and the oxidative/nitrosative stress are altered in postmortem dorsolateral prefrontal cortex of subjects with MDD. The potential modulation induced by the antidepressant treatment on these parameters was also assessed. Thirty MDD subjects (15 antidepressant-free and 15 under antidepressant treatment) were matched for gender and age to 30 controls in a paired design.

Results: No significant changes in TLR-4 expression were detected. An increased expression of the TLR-4 endogenous ligand Hsp70 (+33\%), but not of Hsp60, and the activated forms of mitogen-activated protein kinases (MAPKs) p38 (+ 47\%) and JNK (+ 56\%) was observed in MDD. Concomitantly, MDD subjects present a 45\% decreased expression of DUSP2 (a regulator of MAPKs) and reduced (-21\%) expression of the antioxidant nuclear factor Nrf2. Antidepressant treatment did not modify the changes detected in the group with MDD and actually increased (+ 25\%) the expression of p11, a protein linked with the transport of neurotransmitters and depression.

Conclusion: Data indicate an altered TLR-4 immune response in the brain of subjects with MDD. Additional research focused on the mechanisms contributing to the antidepressant-induced TLR-4 pathway modulation is warranted and could help to develop new treatment strategies for MDD.
\end{abstract}

Keywords: Major depression, Postmortem frontal cortex, Antidepressants, Neuroinflammation, Toll-like receptor 4 pathway, Mitogen-activated protein kinases, Nrf2 pathway

\section{Background}

Many studies are focusing on identifying new etiopathological trails and therapeutic targets of depression. One

\footnotetext{
* Correspondence: jrcaso@med.ucm.es

${ }^{\dagger}$ David Martín-Hernández and Javier R. Caso contributed equally to this work. 'Centro de Investigación Biomédica en Red de Salud Mental (CIBERSAM), Instituto de Salud Carlos III (ISCIII), C/ Monforte de Lemos 3-5, 28029 Madrid, Spain

${ }^{2}$ Departamento de Farmacología y Toxicología, Facultad de Medicina, Universidad Complutense de Madrid, Instituto Universitario de Investigación en Neuroquímica UCM, Avda. Complutense s/n, 28040 Madrid, Spain Full list of author information is available at the end of the article
}

promising development is the apperception of the inflammation, both in the brain and periphery, as an element worth to consider in the pathogenesis of major depressive disorder (MDD). This concept is grounded in the detection of activated inflammatory/immune response in this disease $[1,2]$ both in the brain and in the periphery.

Studies demonstrate that MDD is associated with manifestation of inflammatory markers [3]. Moreover, meta-analyses show increased inflammatory parameters in the blood of patients with MDD [4,5]. Alternatively, patients undergoing cytokine therapy develop depressive

(c) The Author(s). 2018 Open Access This article is distributed under the terms of the Creative Commons Attribution 4.0 International License (http://creativecommons.org/licenses/by/4.0/), which permits unrestricted use, distribution, and 
symptoms [6, 7] and experimental-induced inflammation promotes mood deterioration in healthy subjects [8]. Furthermore, elevated inflammatory markers predict a poorer response to antidepressants $(\mathrm{AD})$ while non-responding patients to $\mathrm{AD}$ show persistently elevated inflammation [9].

Currently, increasing attention is being paid to the potential role of the innate immune system in the pathogenesis of psychiatric diseases, particularly to Toll-like receptors (TLRs) [10]. TLRs trigger a complex proinflammatory cascade that can regulate central nervous system (CNS) homeostasis and even promote pathology [11]. TLRs are expressed not only in immune cells, but also in neurones, astroglia, and resident microglia [12-14]. Their ubiquitous distribution suggests that TLRs play other roles in non-pathogen-associated CNS diseases/injuries, presumably through recognizing endogenous molecules released from damaged tissues, also known as damage-associated molecular patterns (DAMPs) (e.g., heat shock protein Hsp60, Hsp70) [15].

Most of studies have focused on TLR-4, which responds predominantly to lipopolysaccharide (LPS), a component of the outer membrane of Gram-negative bacteria [16]. TLR-4 triggers a transduction pathway (i.e., specific kinases including mitogen-activated protein kinases - MAPKs) resulting in the activation of inflammatory nuclear transcription factors [17]. Many of the MAPK pathway downstream targets are transcription factors that coordinate the transcription of several genes encoding inflammatory mediators, such as cytokines, chemokines, and inducible pro-oxidative enzymes [17].

Despite the considerable evidence suggesting active inflammatory pathways in MDD, confirmation of abnormalities in the postmortem brain of affected subjects has been in some way elusive. Increased cytokine and chemokine gene expression and/or concentrations have been shown in the frontal cortex of MDD subjects [18-21]. Aditionally, altered microglial kynurenines have been described in the cingulate gyrus and hippocampus of MDD [22, 23] and an astrocytic hypertrophy in the anterior cingulate white matter of depressed suicides has been reported [24]. Moreover, a recent study employing dentate gyrus proposes neuroinflammation as crucial in MDD [25].

An important limitation in brain postmortem studies is the possibility that presence of antidepressant treatment could affect the expression and function of cytokines and other inflammatory factors. Most of the previous postmortem studies were performed under experimental conditions that were not designed to control for this factor.

The present work aims to study whether specific elements of intracellular pathways controlling inflammation along with factors related to the restraining of the oxidative/nitrosative stress (i.e., the nuclear factor Nrf2) are altered in postmortem dorsolateral prefrontal cortex of subjects with MDD. In particular, this study has focused on innate immunity-related elements ordinarily understudied and novel parameters such as MAPKs and the antioxidant nuclear factor Nrf2 pathways, respectively. The potential modulation induced by the antidepressant treatment on these parameters was also assessed.

\section{Methods}

\section{Postmortem human brain samples}

Human brain samples from dorsolateral prefrontal cortex (Brodmann's area 9) were obtained at autopsy in the Basque Institute of Legal Medicine, Bilbao, Spain, in compliance with research policies and ethical committees for postmortem brain studies. After a retrospective search for antemortem medical information, 30 Caucasian subjects diagnosed with MDD (DSM-IV, DSM-IV-R, or CIE-10 criteria) were matched for ethnic origin, gender, and age ( \pm 4 years) to 30 control subjects in a paired design. Since non-compliance is frequently observed, the absence or presence of antidepressant drugs was defined according to the toxicological screening at the time of death. Thus, MDD subjects were divided into two groups: subjects antidepressant-free at time of death (AD-free; $n=15$ ) and subjects antidepressant-treated at time of death (AD-treated; $n=15$ ), see Additional file 1 for full details about sample and demographic information.

\section{Preparation of nuclear and cytosolic extracts}

A widely utilized method that provides a high purity nuclear fraction, practically without cytosolic contamination [26] was used (see Additional file 1).

\section{Western blot analysis}

The expression levels of TLR-4, Hsp60, Hsp70, phosphoERK 1/2, phospho-JNK, phospho-p38, p38 $\alpha / \beta$, PI3K, Keap-1, and S100A10 (p11) in cytosolic extracts and the expression levels of DUSP-2, Nrf-2, and p65 (NF-кB subunit) in nuclear extracts from brain samples were analyzed through Western blot (see Additional file 1).

\section{Protein assay}

Protein levels were measured using Bradford method based on the principle of protein-dye binding.

\section{Chemicals and statistical analyses}

Unless otherwise stated, the chemicals were from SigmaAldrich (Spain).

Estimation of the sample size was drawn from previous postmortem studies of neuroinflammation, apoptosis, and oxidative/nitrosative stress in schizophrenia [27] and MDD [28].

Data are expressed as mean \pm SEM. Protein expression data were normalized for respective endogenous control expression ( $\beta$-actin or GAPDH) and relative to a reference standard (pool of control samples), whose expression level 
was defined as $100 \%$. The quantification procedure was repeated three times for each pair of subjects in different gels. The mean value of the different gels was used as a final estimate.

Unpaired two-tailed $t$ test was performed when comparing controls vs MDD and a two-tailed $t$ test with Welch's correction was employed when samples did not have equal standard deviations. After completion of the assays, since the control groups for $\mathrm{AD}$-free and $\mathrm{AD}$-treated subjects did not differ from each other for confounding factors (see Additional file 1: Table S1), they were pooled for further statistical analyses. One-way ANOVA with a Fisher's LSD post hoc test was used to compare between controls and AD-free and AD-treated MDD groups. Data were analyzed using the Brown-Forsythe test to assess the Gaussian distribution. In cases in which data did not follow a Gaussian distribution, a Kruskal-Wallis test followed by post hoc Dunn's test was performed. Correlations were performed to evaluate the influence of the postmortem delay, storage time, and body mass index (BMI). When positive, the results were tested by ANCOVA with the corresponding factor as covariate. In all the cases, a $p$ value $<0.05$ was considered statistically significant.

\section{Results}

The different groups of controls, AD-free and AD-treated subjects at time of death, were comparable attending to gender ratio, age, postmortem delay (PMD), storage time, brain pH RNA integrity number (RIN), and blood ethanol concentrations (Additional file 1: Table S1). BMI was lower in MDD subjects than that in controls (Additional file 1: Table S1). Age at death significantly correlated only with Hsp70 expression $(r=0.54, p=0.004)$. This demographic factor did not influence results due to the matched paired design. BMI showed inverse correlation with p-p38 $(r=-0.35, p=0.007)$, ratio p-p38/total p38 $(r=-0.36, p=0.005)$, and Keap-1 $(r=-0.28, p=$ $0.04)$ expression and positive correlation with NF-kB p65 expression $(r=0.31, p=0.04)$. There was also a positive correlation between PMD and Hsp60 expression ( $r=$ $0.28, p=0.03)$. Ethanol concentrations displayed significant correlation $(r=0.43, p=0.003)$ with S100A10 (p11). Other drugs than antidepressants did not show influence on inflammatory and antioxidant parameters.

\section{Effects of MDD and antidepressant treatment on the expression of TLR-4 and its endogenous ligands Hsp60 and Hsp70}

TLR-4 expression analyses revealed no significant differences between controls and subjects with MDD (Fig. 1a, b).

There were no statistical differences either when the protein expression values of heat shock protein Hsp60 were analyzed (Fig. 1c, d). The results would not change when controlled for PMD effect. Conversely, subjects with
MDD in bulk showed a significant 33\% increase in the Hsp70 protein expression compared with controls (Fig. 1e). When the AD-free and the AD-treated at time of death groups were split, but both groups maintained a trend to show higher values than control group however without statistical significance (Fig. 1f).

\section{Effects of MDD and antidepressant treatment on the expression of MAPKs and the MAPK activity regulator DUSP2}

Subjects with MDD showed an increased expression of the activated (phosphorylated) forms of the extracellular signal-regulated kinases (ERK) $1 / 2(+22 \%, t=2.293$, $p=0.03$ ) and the c-Jun N-terminal kinases (JNK) (+ $56 \%, t=2.468, p=0.02)$ but not the p38 MAPK $(+19 \%$, $t=1.317, p=0.19)$ compared with matched controls (Fig. 2a, c). The activated forms of ERK1/2 and JNK increased in the AD-treated group when compared with the controls. Although phospho-p38 inversely correlates with BMI, ANCOVA demonstrated that controlling for this factor did not influence the results. When considering the ratio between the phosphorylated and the total forms of the proteins, the increase for p-ERK1/2/total ERK1/2 did not reach statistical significance whereas $\mathrm{p}-\mathrm{JNK} /$ total JNK and p-p38/total p38 were clearly higher in MDD than in controls (Fig. 2e). The phosphorylated forms and ratios for p-ERK1/2/total ERK1/2, p-JNK/total JNK, and p-p38/total p38 were selectively increased in AD-treated subjects although did not reach statistical significance in the case of p-ERK1/2/total ERK1/2 ratio (Fig. 2b, d, f). ANCOVA controlling for BMI did not modify p-p38/total p38 ratio results.

Dual-specificity phosphatase 2 (DUSP2) regulates MAPK activity. DUSP2 expression decreased by $45 \%$ in MDD compared with matched controls (Fig. $2 \mathrm{~g}$ ). The statistical difference was retained both in the AD-free at time of death and the AD-treated groups when compared with controls (Fig. 2h).

\section{Effects of MDD and antidepressant treatment on the antioxidant nuclear factor (erythroid 2-derived)-like 2 (Nrf2) pathway}

The PI3K (phosphoinositide 3-kinase) is an activator of the antioxidant transcription factor Nrf2. Subjects with MDD presented an unaltered PI3K expression when compared with controls (Fig. 3a, b).

The Keap-1 (Kelch-like ECH-associated protein 1) is the cytoplasmic inhibitor of Nrf2. Subjects AD-free and AD-treated at time of death showed a non-significant increase in the expression of Keap-1 when compared with controls (Fig. 3c, d). Results of Keap-1 were not modified when controlling for BMI.

Subjects with MDD showed a 21\% decrease of nuclear expression of Nrf2 (Fig. 3e). Statistical significance in the 

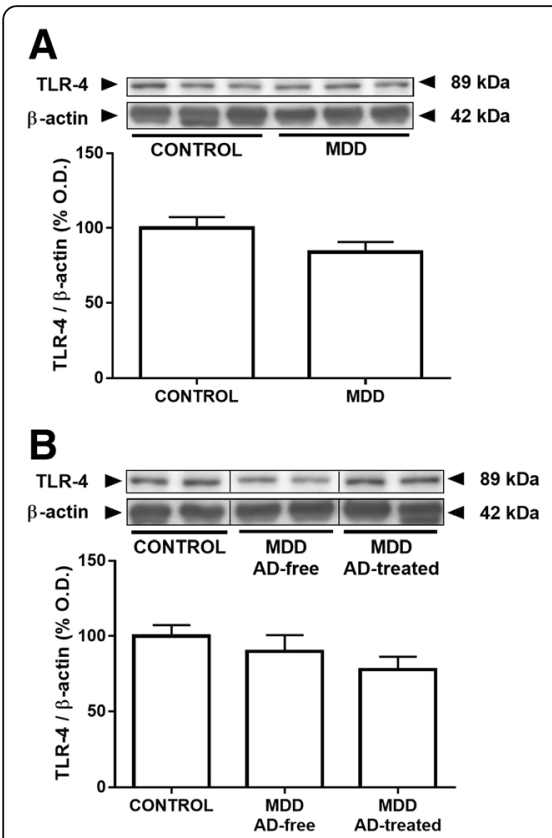
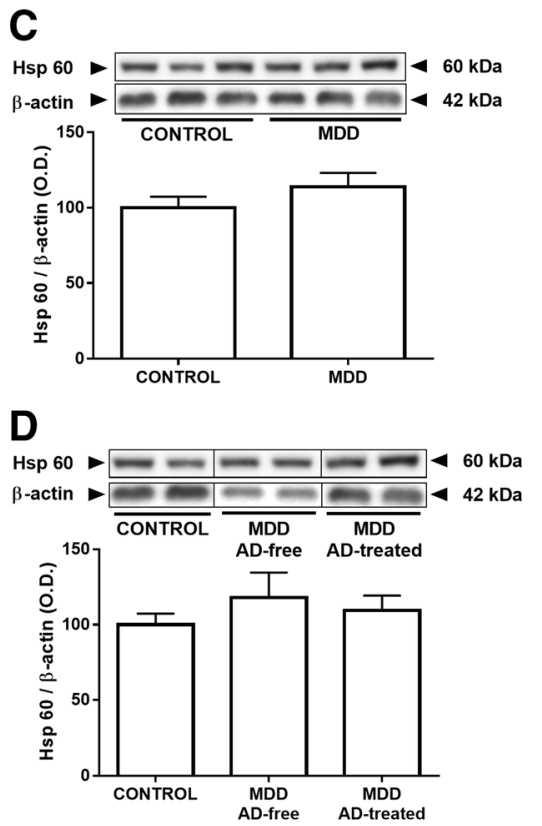

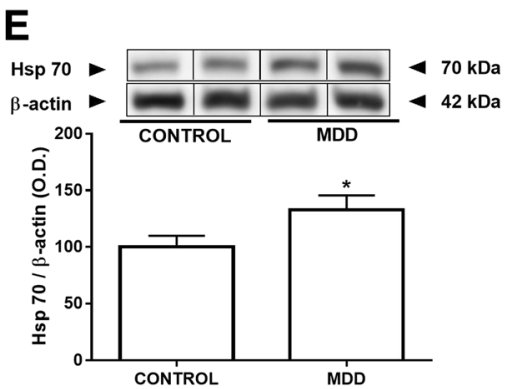

$\mathbf{F}$

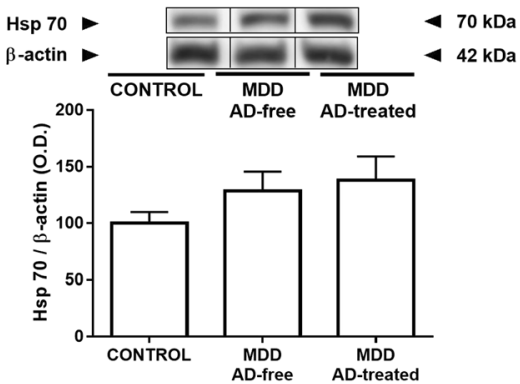

Fig. 1 Effects of MDD and antidepressant treatment on the expression of TLR-4 and its endogenous ligands Hsp60 and Hsp70. There were no differences in the protein expression of TLR-4 among controls and subjects with $\operatorname{MDD}(\mathbf{a}, \mathbf{b})$. There were no statistical differences between groups in the protein expression values of Hsp60 (c, d). Subjects with MDD showed a significant 33\% increase in the Hsp70 protein expression compared with controls (e). The statistical significance disappeared when the AD-free at death and the AD-treated groups were split, but both groups maintained a trend to show higher values than control group (f). The densitometric data of the band of interest were normalized by beta-actin. Some blots were cropped (black lines) for improving the clarity and conciseness of the presentation. Data are means $\pm \mathrm{SEM}$; ${ }^{*} p<0.05$ vs. control; unpaired two-tailed $t$ test was performed when comparing controls vs MDD. One-way ANOVA with a Fisher's LSD post hoc test was used to compare between controls and AD-free and AD-treated MDD groups. See the "Chemicals and statistical analyses" section for more details

analysis of variance was observed only in the AD-treated group, which showed a $31 \%$ decrease of nuclear expression of Nrf2 compared with controls (Fig. 3f).

\section{Effects of MDD and antidepressant treatment on the expression of S100A10 (p11)}

The S100A10 (also known as p11) is a multifactorial protein linked with the transport of neurotransmitters and depression. There were no significant differences between the control and the MDD groups (Fig. 4a). However, when considering MDD subjects according to antidepressant treatment, p11 was elevated in the AD-treated group compared with the AD-free at time of death and the control groups (Fig. 4b). ANCOVA controlling for ethanol concentrations, maintained the significance of p11 increment in MDD.

\section{Effects of MDD and antidepressant treatment on the expression of NF-KB}

The p65 subunit of the transcription factor NF- $\mathrm{kB}$, which controls the transcription of many acute-phase proteins and inflammatory genes [29], was analyzed, showing decreased expression in subjects with MDD (Fig. 4c), both in AD-free $(-46 \%)$ and $\mathrm{AD}$-treated $(-38 \%)$ groups at time of death (Fig. 4d). When results were controlled for BMI of the subjects, which has well-known effects on neuroinflammation [30], ANCOVA analysis failed to show statistical significance $(p=0.09)$.

\section{Discussion}

The present work points to an alteration of inflammatory and oxidative/nitrosative pathways in the brain of subjects with MDD. Different cytoplasmic and nuclear factors involved in neuroinflammatory processes such as constituents of innate immunity response, elements of the antioxidant Nrf2 pathway, and transcription factors were assessed. Data indicate that the overexpression of proinflammatory MAPK pathways is associated with $A D$ treatments, something partially described in in vitro and in vivo experimental settings [31-34] and in postmortem brain of subjects with MDD [28].

In the CNS, the innate immunity receptor TLR-4 is expressed in microglia, neurones, astroglia, oligodendroglia, and cerebral vascular endothelium [35]. Altered TLR-4 signaling has been described in peripheral blood cells and the brain of patients with schizophrenia, bipolar disease, and depression [10]; in postmortem brain of subjects with schizophrenia [27, 36]; and in stress animal models [10]. 
A

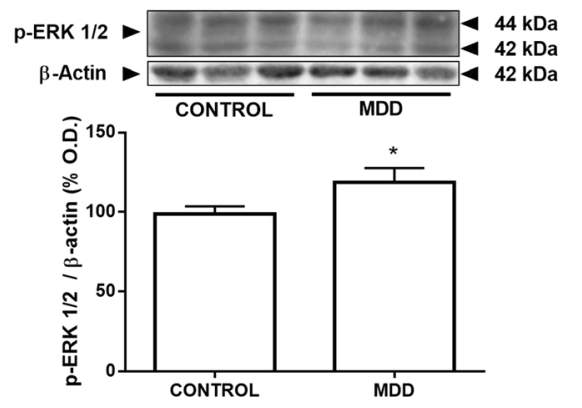

C

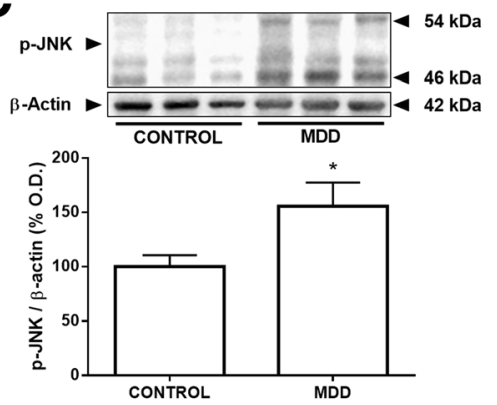

E

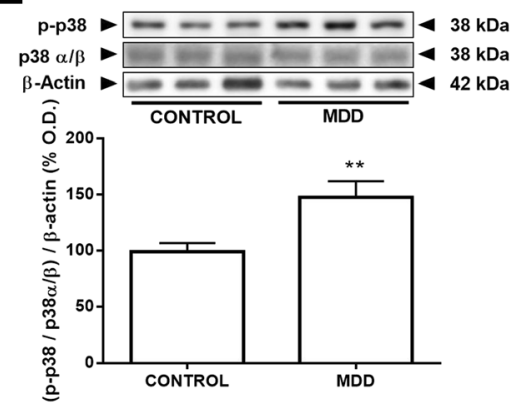

G

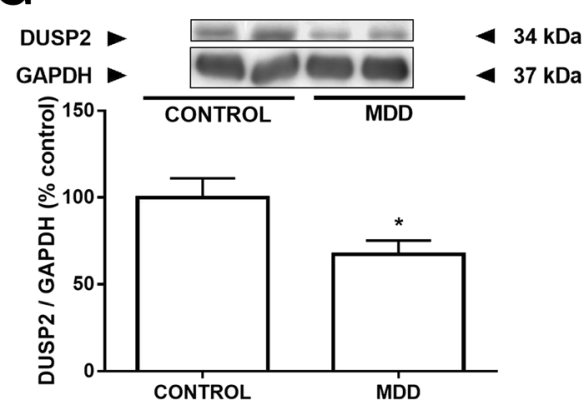

B

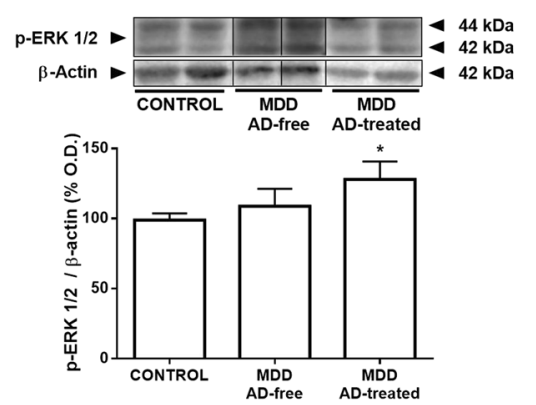

D

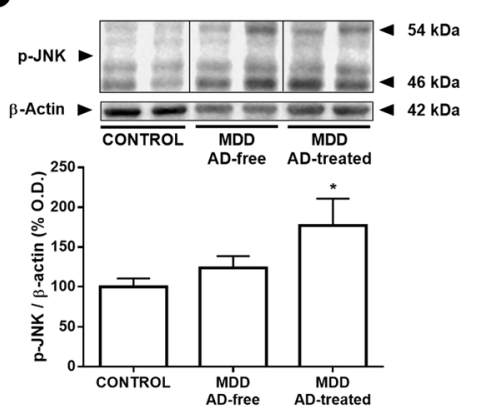

F

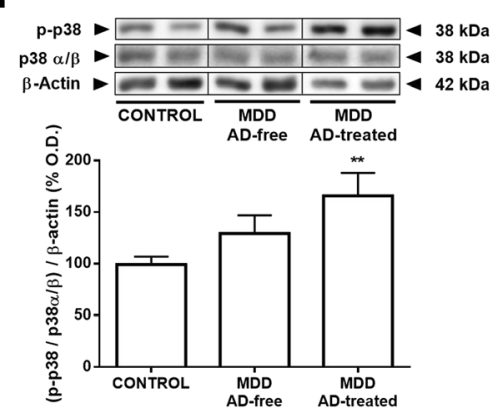

H

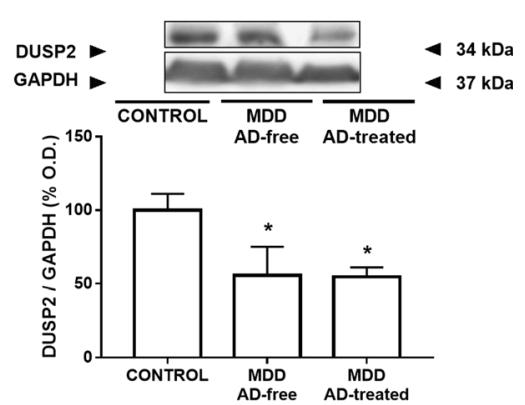

Fig. 2 Effects of MDD and antidepressant treatment on the expression of MAPKs and on the expression of the MAPK activity regulator DUSP2. When considering the phosphorylated forms of the proteins, p-ERK1/2, p-JNK, and the ratio between p-p38/total p38 were clearly higher in MDD than in controls $(\mathbf{a}, \mathbf{c}, \mathbf{e})$. The expression values were selectively increased in AD-treated subjects $(\mathbf{b}, \mathbf{d}$, $\mathbf{f}$. Analysis of covariance controlling for BMl did not modify p-p38/ total p38 ratio results. DUSP2 expression was decreased in MDD compared with matched controls (g), and this statistical difference appeared both in the subjects AD-free at time of death and in AD-treated subjects when compared with controls (h). The densitometric data of the band of interest were normalized by beta-actin. Some blots were cropped (black lines) for improving the clarity and conciseness of the presentation. Data are means \pm SEM; ${ }^{*} p<0.05,{ }^{* *} p<0.01$ vs. control; unpaired two-tailed $t$ test was performed when comparing controls vs MDD. One-way ANOVA with a Fisher's LSD post hoc test was used to compare between controls and AD-free and AD-treated MDD groups. See the "Chemicals and statistical analyses" section for more details 


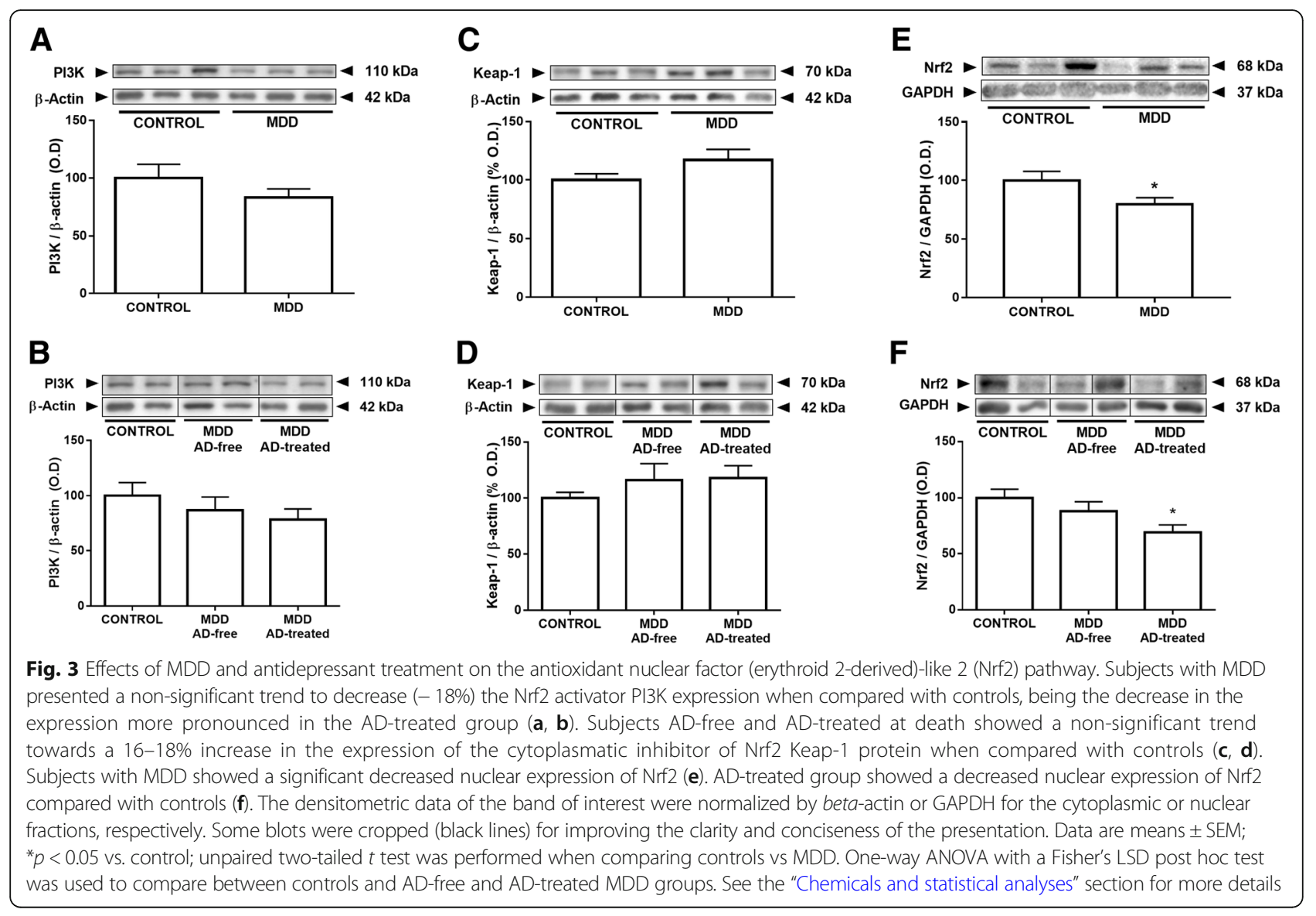

There are two predominant sources of TLR-4 activation: (i) altered gut microbiota [37] and the subsequent dysbiosis through increased intestinal barrier permeability and bacterial translocation present in patients with $\operatorname{MDD}[38,39]$, and (ii) damage-associated molecular patterns (DAMPs) released by non-infective tissue injuries [40]. Among these DAMPs, the heat shock protein Hsp60 and the Hsp70 are well-known stimulators of the TLR-4 [16]. The present results indicate that TLR-4 expression was not noticeably induced in MDD subjects, at least at the moment when the samples were taken. These results also show that the treatment with antidepressants (AD-treated group) tends to decrease TLR-4 expression in the brain. Increased mRNA expression of TLR-4 and later reduction below control levels after AD treatment have been recently described in peripheral blood cells of patients with MDD [41]. In the dorsolateral prefrontal cortex of depressed subjects, TLR-4 increased mRNA expression has been previously found together with an unaltered TLR-4 protein expression in non-suicide victims and elevation in suicide victims [42]. In this previous study, although the influence of AD treatment was considered, a controlled design for this purpose was not included which prevents from drawing definitive conclusions. Consistent with the hypothesis of a DAMP activation of TLR-4 pathways in MDD, evidence of enhanced Hsp70 protein expression is here provided. No differences in Hsp70 expression are observed between AD-free at time of death and AD-treated subjects, suggesting that elevation of the endogenous ligand Hsp70 is rather associated to the disorder and that downregulation of the TLR-4 receptor after AD treatment represents the compensatory effect against the hyperactive pathway. An equivalent observation has been found for the Hsp70 family member GRP78 in postmortem brain of depressed suicide victims [43]. Whether increased Hsp70 expression represents a dysfunction originated in the periphery or a primary CNS impairment remains to be elucidated.

The MAPKs are downstream elements activated after TLR-4 stimulation $[17,44]$. The MAPK pathways are implicated in a wide range of signaling cascades wherein various extracellular stimuli induce inflammation. It has been shown that MAPKs can play as well an important role in the regulation of the NF- $\mathrm{KB}$ pathway [45]. Moreover, proinflammatory cytokines can increase, through activation of signaling pathways that include MAPKs, the expression and function of monoamine transporters $[46,47]$, the most important target for antidepressant 

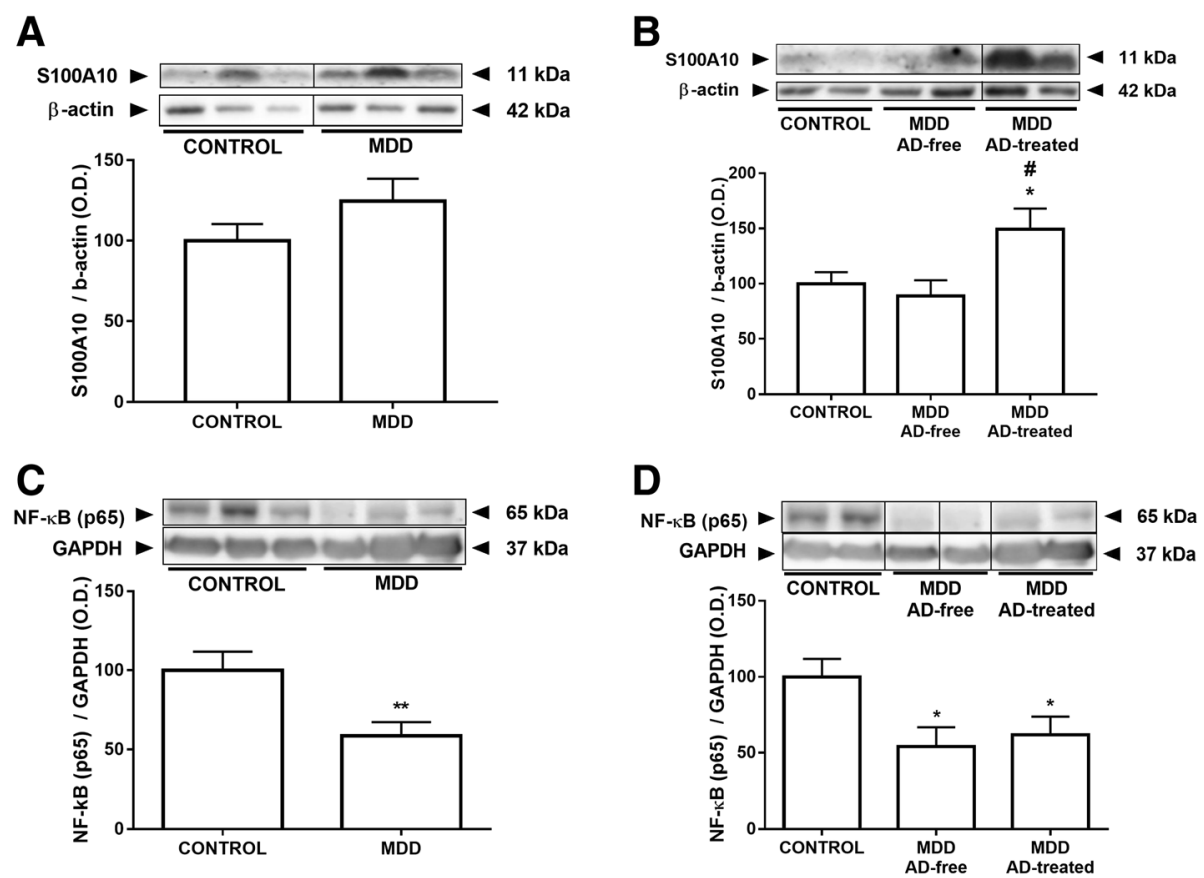

Fig. 4 Effects of MDD and AD treatment on the expression of S100A10 (p11) and NF-KB. There were no significant differences in the p11 expression between control and MDD groups (a). p11 was elevated in the AD-treated at death group compared with the AD-free at death and the control groups (b). The p65 subunit of the transcription factor NF-KB expression was decreased in patients with MDD compared with controls (c) and when AD-free and AD-treated at death groups were compared with matched controls (d). The densitometric data of the band of interest were normalized by beta-actin or GAPDH for the cytoplasmatic or nuclear fractions, respectively. Some blots were cropped (black lines) for improving the clarity and conciseness of the presentation. Data are means $\pm \mathrm{SEM} ;{ }^{*} p<0.05,{ }^{* *} p<0.01 \mathrm{vs}$. control; ${ }^{*} p<0.05 \mathrm{vs}$. AD-free; unpaired two-tailed $t$ test was performed when comparing controls vs MDD. One-way ANOVA with a Fisher's LSD post hoc test was used to compare between controls and AD-free and AD-treated MDD groups. See the "Chemicals and statistical analyses" section for more details

drugs. In this context, genes related to MAPK pathways seem to be upregulated by chronic antidepressant treatment [33]. Data suggest increased expression of the activated forms of ERK1/2, JNK, and p38 proteins in MDD. When the MDD group is evaluated considering the presence of $\mathrm{AD}$ drugs, the elevation is mainly observed in AD-treated subjects. Thus, the presence of AD treatment might be associated with an overactivation of MAPK pathways.

Changes in basal ERK activation produce structural changes in neurones responsible for phenotypes of mood disorders [48]. ERK has been related to the regulation of gene expression and protein synthesis, shifts in the cellular structure and metabolism, cell differentiation and apoptosis. All these regulatory effects might play a role in the impairments of neural plasticity, neurogenesis, and cellular resilience described in the brain of subjects with depression [49]. Furthermore, the effectiveness of drugs such as mood stabilizers and antidepressants partially depends on their capacity to stimulate ERK in the CNS $[49,50]$. In postmortem frontal cortex of suicide victims with MDD, mainly in AD-free conditions, a decreased expression and activation of total ERK1/2 has been previously described [51]. Another study in postmortem brain has found unaltered total ERK $1 / 2$ protein expression with decrease of active p-ERK $1 / 2$ forms that are normalized under AD treatment [28]. These data are quite consistent with results in rats exposed to stress where chronic fluoxetine reversed the lower p-ERK1/2 protein expression in the hippocampus and frontal cortex [32]. Accordingly, in the present study, subjects $\mathrm{AD}$-free at time of death show a non-significant dropping tendency $(-26 \%)$ of total ERK1/2 expression when compared to controls whereas only a $7 \%$ reduction is obtained in AD-treated (data not shown). On the other hand, the expression of p-ERK1/2 (activated) form and p-ERK1/2/total ERK1/2 ratio is markedly increased in MDD subjects under AD treatment. Thus, AD treatment seems to promote expression of activated ERK forms, possibly in an attempt to recover the plasticity and counteract the negative cellular effects associated with the reduced expression of ERK observed in the disorder.

An increased expression of the activated forms of JNK and p38 in subjects with MDD is shown here. Importantly, their expression follows the same pattern as the expression of ERK, being higher in the AD-treated group. 


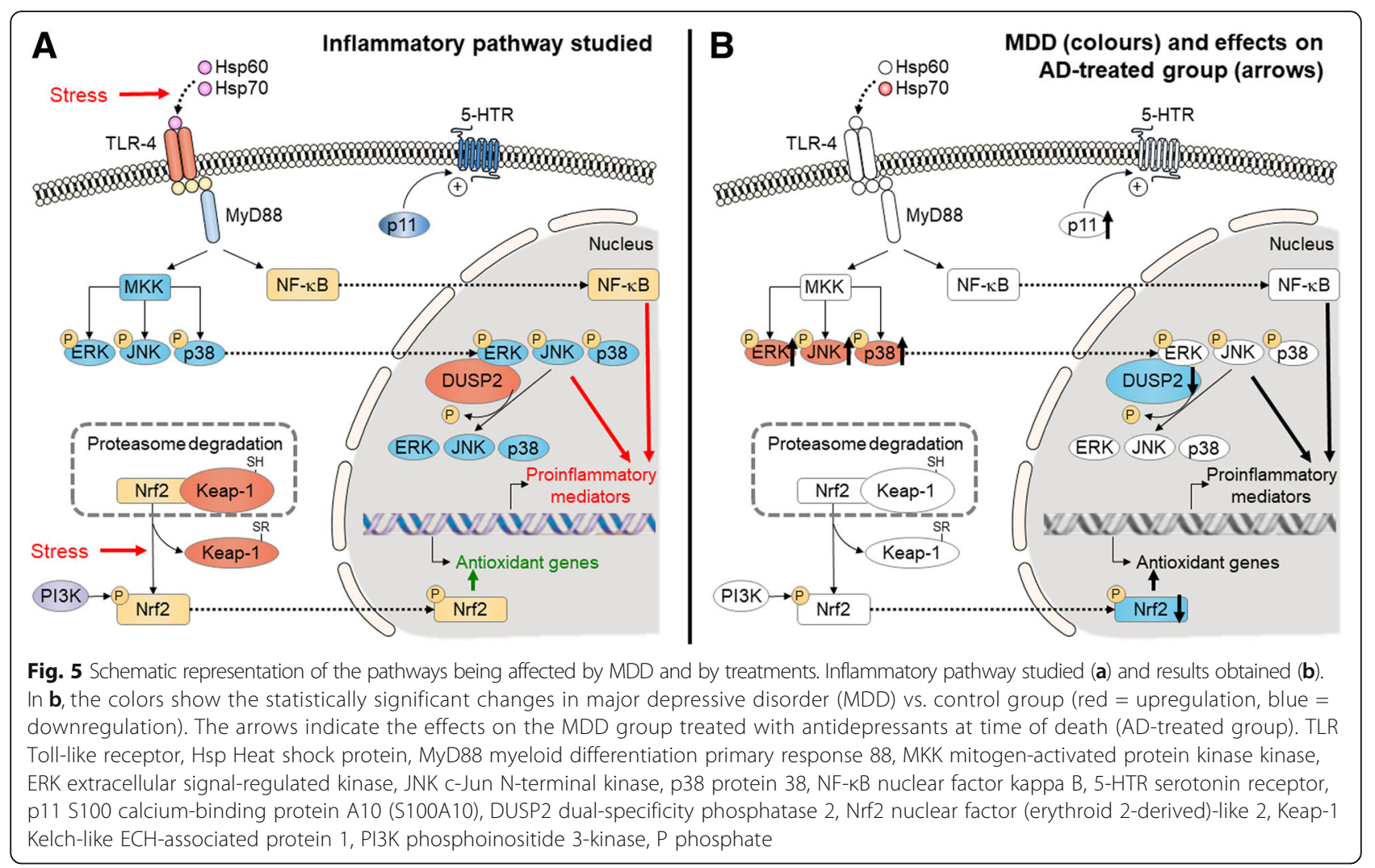

Both p38 and JNK have been linked to the pathophysiology of depression [52]. Specifically, p38 has been related to MDD because of its proinflammatory actions and its stimulatory activity on the expression and function of the serotonin transporter [2]. JNK plays a critical role in the regulation of $\mathrm{T}$ lymphocyte differentiation and cytokine production [52]. Inhibition of the JNK pathway in animal models of depression [53] and activation of p38-dependent pathways by the antidepressant amitriptyline [54] have been previously described. Further research is needed to evaluate the role of these MAPKs in MDD and their modulation by AD drugs.

Dual-specificity phosphatases (DUSPs) are a heterogeneous group of protein phosphatases that regulate activity of MAPKs through dephosphorylation (deactivation) and serve to control the MAPK subcellular localization [55]. DUSP2 displays substrate specificity for ERK, p38, and JNK. In fact, preclinical in vivo studies revealed that DUSP2 is a negative regulator of JNK activity and positive regulator of immune responses via cross-talk between JNK and ERK [56]. The present results show a decrease in DUSP2 expression in the MDD group, both in subjects AD-free and AD-treated at time of death. This result could explain, at least partially, the trend towards enhanced MAPK expression in MDD.
Nrf2 is a main cellular defense pathway activated by oxidative stress, leading to production of target antioxidants [57]. A role for the Nrf2 pathway in experimental models of depression has been described $[58,59]$. Thus, Nrf2 null mice are more vulnerable to develop depression-like phenotypes [60]. Indeed, Nrf2 has been proposed as a potential therapeutic target worth to consider in future studies [61]. Our data indicate a decrease of the Nrf2 pathway in subjects with MDD. This decrease would be in agreement with the proposed role of Nrf2 in the pathophysiology of depression, being involved in the enhanced oxidative/nitrosative stress described in this disorder [62]. Antidepressant treatment affected the nuclear expression of Nrf2. Previous preclinical studies showed that antidepressants modulate this pathway and that this effect differs depending of the CNS region analyzed [59]. Moreover, there are results in human monocytic U-937 cells, suggesting that the effects of $\mathrm{AD}$ on the expression of antioxidant enzymes are dependent on the duration of the treatment and even can have pro-oxidant consequences [63]. Here, AD treatments do not reverse the trend to reduction observed in AD-free at time of death subjects and even could be negatively affecting the Nrf2 protective pathway. The possibility that both groups of subjects could represent a population with resistance to AD should 
bear in mind due to the presence of suicide as the main cause of the death. The downward trend in the expression of the Nrf2 activator PI3K and increased expression of the Nrf2 inhibitor Keap-1 both in $\mathrm{AD}$-free and $\mathrm{AD}$-treated populations at time of death is congruent with the nuclear reduction of this transcription factor.

The calcium-binding protein $\mathrm{p} 11$ is linked with the transport of neurotransmitters and depression and data from cell lines confirm that it is regulated by immunological parameters like TLR-4 [64]. Administration of proinflammatory cytokines stimulates the formation of p11 through unknown mechanisms [65]. Experimental data indicate that p11 knockout mice display a depressive-like phenotype whereas p11 overexpressing mice display antidepressant-like responses [66]. Moreover, p11 is upregulated in the CNS after chronic antidepressant treatments. Once upregulated, p11 increases the expression of 5-HT receptors on the plasma membrane, providing an enhancer mechanism for antidepressant actions [67]. Our results show a specific augmentation of $\mathrm{p} 11$ protein expression in the AD-treated group compared with the $\mathrm{AD}$-free group at time of death and controls. The data agree with prior results in animal models of depression and humans suffering with MDD where decreased p11 expression was reported [66]. However, it is the first evidence showing that $\mathrm{AD}$ treatment may result in increased p11 protein expression reported for MMD subjects.

$\mathrm{NF}-\mathrm{kB}$ is a nuclear factor that controls the transcription of many acute-phase proteins and inflammatory genes, being one of the early events in the stress-induced inflammatory response in CNS [26]. Although hyperactive $\mathrm{NF}-\mathrm{KB}$ in peripheral cells has been considered a fingerprint of stress and mood disorders, in animals under chronic mild stress conditions, small reductions of NF- $\mathrm{kB}$ p65 mRNA and protein expression and activity have been described [26]. The present study suggests a similar tendency in the human brain. However, control subjects exhibited a higher BMI, a physical parameter that displayed positive relationship with NF- $\kappa B$ expression. Therefore, larger studies of controlled populations for BMI are necessary to establish definitive conclusions on the status of $\mathrm{NF}-\mathrm{kB}$ in the brain of subjects with MDD. Besides, NF- $\mathrm{kB}$ is not only a main regulator of the inflammatory response but is also an essential regulatory element of cell survival, controlling key processes such as neuroprotection, neuronal transmission, and plasticity [68]. Once again, more studies are warranted in order to fully understand the role of NF- $\kappa B$ in this particular setting.

Suicide represents a special cause of death that may act as a confounding factor in studies using postmortem brain samples from subjects with psychiatric disorders. Since suicide is highly prevalent in depressive disorders, the neurobiology underlying suicide might mask some results in MDD studies. Therefore, the assumption that description of inflammation pathways altered in the brain of depressed suicide victims can automatically be extended to depressed non-suicide victims is potentially faulty. In fact, case points of differences between suicide and non-suicide depressed subjects for TLR-4 [42] and microglial density [69] have been published. Moreover, MDD subjects died by suicide and under confirmed antidepressant treatment, as herein presented, should be considered more representative of resistant than standard depression. Although suicide was the cause of death for an important number of MDD subjects in the present study, opposite results for TLR- 4 and NF- $\mathrm{kB}$ expression have been recently described in a schizophrenia population mainly dead by suicide [27]. Therefore, at least some of the changes herein observed in the postmortem brain of MDD subjects should be associated to depression and antidepressant treatment rather than to suicide behavior. About $4 \%$ of depressed individuals die by suicide, being the risk greater in males [70]. Although depression is more prevalent in female, this factor could explain the higher presence of male subjects in the present study.

The fact that selection of MDD subjects was performed based on clinical records of subjects could be interpreted as a study limitation. In fact, the use of antemortem diagnosis versus postmortem retrospective methodologies based on psychological autopsy is still a matter of controversy [71]. The psychopathological condition of MDD and control subjects at time of death represents also a limitation of this type of studies performed in outpatients who die by sudden and unexpected causes. On the other hand, although control subjects did not show clinical records of neurological or psychiatric disorders, including drug abuse, the possibility of presence of potential non-diagnosed subjects among controls is not completely ruled out. For a better interpretation of the alterations caused by MDD and the actions of antidepressant treatments see Fig. 5.

\section{Conclusions}

Our results show an increase in Hsp70, an endogenous ligand of the TLR- 4 and in the products of the TLR- 4 activation such as MAPKs in postmortem dorsolateral prefrontal cortex of subjects with MDD. An important effect to consider is the influence of the pharmacological treatment with $\mathrm{AD}$ on MAPK expression. Further research focused on the mechanisms that contribute to this modulation is essential and could help developing new treatment strategies for MDD.

Results also show for the first time in the brain of MDD subjects that the disorder is affecting the antioxidant Nrf2 pathway. In particular, a decrease in the antioxidant factor Nrf2 is observed. Complementary, AD treatment induces 
an increase of p11 that could be boosting the expression of 5-HT receptors.

The data seem to point out to the presence of an altered innate immune response in the brain of subjects with MDD, in whom the TLR4 pathway could be one of the main elements influenced. However, Hsp70 also binds to other TLRs as well as other receptors and therefore additional studies are required to explicitly confirm TLR-4 involvement. Similarly, further research is necessary to ascertain the condition of the innate immune system in the MDD and attending especially to the potential confounding factor of antidepressant treatment.

\section{Additional file}

Additional file 1: Supplemental Material. (DOC $71 \mathrm{~kb}$ )

\section{Abbreviations}

AD: Antidepressants; BMI: Body mass index; CNS: Central nervous system; DAMPs: Damage-associated molecular patterns; DUSP2: Dual-specificity phosphatase 2; ERK1/2: Extracellular signal-regulated kinases 1/2; Hsp: Heat shock protein; JNK: c-Jun N-terminal kinases; Keap-1: Kelch-like ECH-associated protein 1; LPS: Lipopolysaccharide; MAPKs: Mitogen-activated protein kinases; MDD: Major depressive disorder; NF-kB: Nuclear factor kappa B; Nrf2: Nuclear factor (erythroid 2-derived)-like 2; PI3K: Phosphoinositide 3-kinase; PMD: Postmortem delay; RIN: RNA integrity number; TLR: Toll-like receptor

\section{Acknowledgements}

The authors thank the staff members of the Basque Institute of Legal Medicine (Bilbao) for their cooperation in this work.

\section{Funding}

This work was supported by the Instituto de Salud Carlos III and Spanish Ministry of Economy, Industry and Competitiveness (MINECO) through the Plan Estatal de I+D+i 2013-2016 (FIS-PI13/01102 and SAF2016-75500-R to JCL), the Agencia Estatal de Investigación (AEI) and Fondo Europeo de Desarrollo Regional (FEDER) (SAF2017-83053-R to JRC), the Basque Government (IT-616-13), CIBERSAM and the EDR Funds. JRC and BGB are Ramón y Cajal fellows (MINECO).

\section{Availability of data and materials}

The data that support the findings of this study are available from the corresponding author upon reasonable request.

\section{Authors' contributions}

$\mathrm{DMH}$ performed the experiments and analyzed the data. JRC conceived and designed the experiments, interpreted the data, and wrote the manuscript. JJM analyzed and interpreted the data and wrote the manuscript. LFC, JLMM, and BGB interpreted the data and reviewed the manuscript. JCL conceived and designed the experiments, interpreted the data, and reviewed the manuscript. All authors read and approved the final version of the manuscript.

\section{Ethics approval and consent to participate}

Human brain samples were collected at autopsy in the Basque Institute of Legal Medicine according to legal regulation for postmortem studies (Royal Decree 1716/2011). Samples were stored in a brain repository (Register C35 in the Instituto de Salud Carlos III). The study was approved by the Ethics Committee of the Complutense University of Madrid (project's reference PI13/01102).

\section{Consent for publication}

Not applicable.

\section{Competing interests}

The authors declare that they have no competing interests.

\section{Publisher's Note}

Springer Nature remains neutral with regard to jurisdictional claims in published maps and institutional affiliations.

\section{Author details}

${ }^{1}$ Centro de Investigación Biomédica en Red de Salud Mental (CIBERSAM), Instituto de Salud Carlos III (ISCIII), C/ Monforte de Lemos 3-5, 28029 Madrid, Spain. ${ }^{2}$ Departamento de Farmacología y Toxicología, Facultad de Medicina, Universidad Complutense de Madrid, Instituto Universitario de Investigación en Neuroquímica UCM, Avda. Complutense s/n, 28040 Madrid, Spain. ${ }^{3}$ Instituto de Investigación Sanitaria Hospital 12 de Octubre (Imas12), Avda. de Córdoba, s/n, 28041 Madrid, Spain. ${ }^{4}$ Departamento de Farmacología, Universidad del País Vasco, UPV/EHU, B. Sarriena s/n, 48940 Leioa, Bizkaia, Spain. ${ }^{5}$ Instituto de Investigación Sanitaria Biocruces, Plaza de Cruces s/n, 48903 Barakaldo, Bizkaia, Spain.

Received: 9 March 2018 Accepted: 28 August 2018

Published online: 04 September 2018

\section{References}

1. Dantzer R, O'Connor JC, Freund GG, Johnson RW, Kelley KW. From inflammation to sickness and depression: when the immune system subjugates the brain. Nat Rev Neurosci. 2008;9:46-56.

2. Haroon E, Raison CL, Miller AH. Psychoneuroimmunology meets neuropsychopharmacology: translational implications of the impact of inflammation on behavior. Neuropsychopharmacology. 2012;37:137-62.

3. Morris AA, Zhao L, Ahmed Y, Stoyanova N, De Staercke C, Hooper WC, Gibbons G, Din-Dzietham R, Quyyumi A, Vaccarino V. Association between depression and inflammation--differences by race and sex: the META-Health study. Psychosom Med. 2011;73:462-8.

4. Dowlati Y, Herrmann N, Swardfager W, Liu H, Sham L, Reim EK, Lanctot KL. A meta-analysis of cytokines in major depression. Biol Psychiatry. 2010;67:446-57.

5. Haapakoski R, Mathieu J, Ebmeier KP, Alenius H, Kivimaki M. Cumulative metaanalysis of interleukins 6 and 1beta, tumour necrosis factor alpha and C-reactive protein in patients with major depressive disorder. Brain Behav Immun. 2015;49: 206-15.

6. Raison $\mathrm{CL}$, Capuron L, Miller AH. Cytokines sing the blues: inflammation and the pathogenesis of depression. Trends Immunol. 2006;27:24-31.

7. Udina M, Hidalgo D, Navines R, Forns X, Sola R, Farre M, Capuron L, Vieta E, Martin-Santos R. Prophylactic antidepressant treatment of interferon-induced depression in chronic hepatitis C: a systematic review and meta-analysis. J Clin Psychiatry. 2014;75:e1113-21.

8. Engler H, Brendt P, Wischermann J, Wegner A, Rohling R, Schoemberg T, Meyer U, Gold R, Peters J, Benson S, Schedlowski M. Selective increase of cerebrospinal fluid IL-6 during experimental systemic inflammation in humans: association with depressive symptoms. Mol Psychiatry. 2017;22:1448-54.

9. Strawbridge R, Arnone D, Danese A, Papadopoulos A, Herane Vives A, Cleare AJ. Inflammation and clinical response to treatment in depression: a meta-analysis. Eur Neuropsychopharmacol. 2015;25:1532-43.

10. Garcia-Bueno B, Caso JR, Madrigal JL, Leza JC. Innate immune receptor Tolllike receptor 4 signalling in neuropsychiatric diseases. Neurosci Biobehav Rev. 2016;64:134-47.

11. Medzhitov R. Toll-like receptors and innate immunity. Nat Rev Immunol. 2001;1:135-45.

12. Crack PJ, Bray PJ. Toll-like receptors in the brain and their potential roles in neuropathology. Immunol Cell Biol. 2007;85:476-80.

13. Garate I, Garcia-Bueno B, Madrigal JL, Caso JR, Alou L, Gomez-Lus ML, Mico JA, Leza JC. Stress-induced neuroinflammation: role of the Toll-like receptor4 pathway. Biol Psychiatry. 2013;73:32-43.

14. Hanke ML, Kielian T. Toll-like receptors in health and disease in the brain: mechanisms and therapeutic potential. Clin Sci (Lond). 2011;121: 367-87.

15. Piccinini AM, Midwood KS. DAMPening inflammation by modulating TLR signalling. Mediat Inflamm. 2010;2010 https://doi.org/10.1155/2010/672395.

16. Takeda K, Kaisho T, Akira S. Toll-like receptors. Annu Rev Immunol. 2003;21: 335-76.

17. Guha M, Mackman N. LPS induction of gene expression in human monocytes. Cell Signal. 2001;13:85-94.

18. Dean B, Tawadros N, Scarr E, Gibbons AS. Regionally-specific changes in levels of tumour necrosis factor in the dorsolateral prefrontal cortex obtained postmortem from subjects with major depressive disorder. J Affect Disord. 2010;120:245-8. 
19. Shelton RC, Claiborne J, Sidoryk-Wegrzynowicz M, Reddy R, Aschner M, Lewis DA, Mirnics K. Altered expression of genes involved in inflammation and apoptosis in frontal cortex in major depression. Mol Psychiatry. 2011;16:751-62.

20. Thomas AJ, Ferrier IN, Kalaria RN, Woodward SA, Ballard C, Oakley A, Perry $\mathrm{RH}, \mathrm{O}$ 'Brien JT. Elevation in late-life depression of intercellular adhesion molecule-1 expression in the dorsolateral prefrontal cortex. Am J Psychiatry. 2000;157:1682-4

21. Torres-Platas SG, Cruceanu C, Chen GG, Turecki G, Mechawar N. Evidence for increased microglial priming and macrophage recruitment in the dorsal anterior cingulate white matter of depressed suicides. Brain Behav Immun. 2014;42:50-9.

22. Steiner J, Walter M, Gos T, Guillemin GJ, Bernstein HG, Sarnyai Z, Mawrin C, Brisch R, Bielau H, Meyer zu Schwabedissen L, et al. Severe depression is associated with increased microglial quinolinic acid in subregions of the anterior cingulate gyrus: evidence for an immune-modulated glutamatergic neurotransmission? J Neuroinflammation. 2011;8:94.

23. Busse M, Busse S, Myint AM, Gos T, Dobrowolny H, Muller UJ, Bogerts B, Bernstein $\mathrm{HG}$, Steiner J. Decreased quinolinic acid in the hippocampus of depressive patients: evidence for local anti-inflammatory and neuroprotective responses? Eur Arch Psychiatry Clin Neurosci. 2015;265:321-9.

24. Torres-Platas SG, Hercher C, Davoli MA, Maussion G, Labonte B, Turecki G, Mechawar N. Astrocytic hypertrophy in anterior cingulate white matter of depressed suicides. Neuropsychopharmacology. 2011;36:2650-8.

25. Mahajan GJ, Vallender EJ, Garrett MR, Challagundla L, Overholser JC, Jurjus G, Dieter L, Syed M, Romero DG, Benghuzzi H, Stockmeier CA. Altered neuro-inflammatory gene expression in hippocampus in major depressive disorder. Prog Neuro-Psychopharmacol Biol Psychiatry. 2018, 82:177-86.

26. Garate I, Garcia-Bueno B, Madrigal JL, Bravo L, Berrocoso E, Caso JR, Mico JA, Leza JC. Origin and consequences of brain Toll-like receptor 4 pathway stimulation in an experimental model of depression. J Neuroinflammation. 2011;8:151.

27. García-Bueno B, Gassó P, MacDowell KS, Callado LF, Mas S, Bernardo M, Lafuente A, Meana JJ, Leza JC. Evidence of activation of Toll-like receptor-4 proinflammatory pathway in schizophrenia. J Psychiatry Neurosci. 2016;41:E46-55.

28. Garcia-Fuster MJ, Diez-Alarcia R, Ferrer-Alcon M, La Harpe R, Meana JJ, GarciaSevilla JA. FADD adaptor and PEA-15/ERK1/2 partners in major depression and schizophrenia postmortem brains: basal contents and effects of psychotropic treatments. Neuroscience. 2014;277:541-51.

29. Mitchell S, Vargas J, Hoffmann A. Signaling via the NFkappaB system. Wiley Interdiscip Rev Syst Biol Med. 2016;8:227-41

30. Lauridsen JK, Olesen RH, Vendelbo J, Hyde TM, Kleinman JE, Bibby BM, Brock B, Rungby J, Larsen A. High BMI levels associate with reduced mRNA expression of IL10 and increased mRNA expression of iNOS (NOS2) in human frontal cortex. Transl Psychiatry. 2017;7:e1044

31. Chou CT, He S, Jan CR. Paroxetine-induced apoptosis in human osteosarcoma cells: activation of p38 MAP kinase and caspase-3 pathways without involvement of [Ca2+]i elevation. Toxicol Appl Pharmacol. 2007;218:265-73.

32. Cui J, Yang K, Yu X, Wang JL, Li J, Zhang Y, Li H. Chronic fluoxetine treatment upregulates the activity of the ERK1/2-NF-kappaB signaling pathway in the hippocampus and prefrontal cortex of rats exposed to forced-swimming stress. Med Princ Pract. 2016;25:539-47.

33. Lopez JP, Fiori LM, Cruceanu C, Lin R, Labonte B, Cates HM, Heller EA, Vialou V, Ku SM, Gerald C, et al. MicroRNAs 146a/b-5 and 425-3p and 24-3p are markers of antidepressant response and regulate MAPKWnt-system genes. Nat Commun. 2017;8:15497.

34. Mercier G, Lennon AM, Renouf B, Dessouroux A, Ramauge M, Courtin F, Pierre M. MAP kinase activation by fluoxetine and its relation to gene expression in cultured rat astrocytes. J Mol Neurosci. 2004;24:207-16.

35. Vargas-Caraveo A, Sayd A, Maus SR, Caso JR, Madrigal JLM, GarcíaBueno B, Leza JC. Lipopolysaccharide enters the rat brain by a lipoprotein-mediated transport mechanism in physiological conditions. Sci Rep. 2017;7:13113.

36. MacDowell KS, Pinacho R, Leza JC, Costa J, Ramos B, Garcia-Bueno B. Differential regulation of the TLR4 signalling pathway in post-mortem prefrontal cortex and cerebellum in chronic schizophrenia: relationship with SP transcription factors. Prog Neuro-Psychopharmacol Biol Psychiatry. 2017; 79:481-92.

37. Zheng P, Zeng B, Zhou C, Liu M, Fang Z, Xu X, Zeng L, Chen J, Fan S, Du X, et al. Gut microbiome remodeling induces depressive-like behaviors through a pathway mediated by the host's metabolism. Mol Psychiatry. 2016;21:786-96.
38. Berk M, Williams L, Jacka FN, O'Neil A, Pasco JA, Moylan S, Allen NB, Stuart AL, Hayley AC, Byrne ML, Maes M. So depression is an inflammatory disease, but where does the inflammation come from? BMC Med. 2013;11:200.

39. Maes M, Kubera M, Leunis JC, Berk M. Increased IgA and IgM responses against gut commensals in chronic depression: further evidence for increased bacterial translocation or leaky gut. J Affect Disord. 2012;141:55-62.

40. Thundyil J, Lim KL. DAMPs and neurodegeneration. Ageing Res Rev. 2015; 24:17-28.

41. Hung YY, Huang KW, Kang HY, Huang GY, Huang TL. Antidepressants normalize elevated Toll-like receptor profile in major depressive disorder. Psychopharmacology. 2016;233:1707-14.

42. Pandey GN, Rizavi HS, Ren X, Bhaumik R, Dwivedi Y. Toll-like receptors in the depressed and suicide brain. J Psychiatr Res. 2014;53:62-8.

43. Bown C, Wang JF, MacQueen G, Young LT. Increased temporal cortex ER stress proteins in depressed subjects who died by suicide. Neuropsychopharmacology. 2000;22:327-32.

44. Konat GW, Kielian T, Marriott I. The role of Toll-like receptors in CNS response to microbial challenge. J Neurochem. 2006;99:1-12.

45. Schmitz ML, Bacher S, Kracht M. I kappa B-independent control of NF-kappa B activity by modulatory phosphorylations. Trends Biochem Sci. 2001;26: 186-90.

46. Moron JA, Zakharova I, Ferrer JV, Merrill GA, Hope B, Lafer EM, Lin ZC, Wang JB, Javitch JA, Galli A, Shippenberg TS. Mitogen-activated protein kinase regulates dopamine transporter surface expression and dopamine transport capacity. J Neurosci. 2003;23:8480-8.

47. Zhu CB, Blakely RD, Hewlett WA. The proinflammatory cytokines interleukin1 beta and tumor necrosis factor-alpha activate serotonin transporters. Neuropsychopharmacology. 2006;31:2121-31.

48. Einat H, Manji HK. Cellular plasticity cascades: genes-to-behavior pathways in animal models of bipolar disorder. Biol Psychiatry. 2006;59:1160-71.

49. Shiflett MW, Balleine BW. Contributions of ERK signaling in the striatum to instrumental learning and performance. Behav Brain Res. 2011;218:240-7.

50. Gourley SL, Wu FJ, Kiraly DD, Ploski JE, Kedves AT, Duman RS, Taylor JR. Regionally specific regulation of ERK MAP kinase in a model of antidepressant-sensitive chronic depression. Biol Psychiatry. 2008;63:353-9.

51. Dwivedi Y, Rizavi HS, Roberts RC, Conley RC, Tamminga CA, Pandey GN. Reduced activation and expression of ERK1/2 MAP kinase in the postmortem brain of depressed suicide subjects. J Neurochem. 2001;77:916-28.

52. Galeotti N, Ghelardini C. Regionally selective activation and differential regulation of ERK, JNK and p38 MAP kinase signalling pathway by protein kinase C in mood modulation. Int J Neuropsychopharmacol. 2012;15:78193.

53. Clarke M, Pentz R, Bobyn J, Hayley S. Stressor-like effects of c-Jun N-terminal kinase (JNK) inhibition. PLoS One. 2012;7:e44073.

54. Morioka N, Suekama K, Zhang FF, Kajitani N, Hisaoka-Nakashima K, Takebayashi M, Nakata Y. Amitriptyline up-regulates connexin43-gap junction in rat cultured cortical astrocytes via activation of the p38 and c-Fos/AP-1 signalling pathway. Br J Pharmacol. 2014;171:2854-67.

55. Patterson Kl, Brummer T, O'Brien PM, Daly RJ. Dual-specificity phosphatases: critical regulators with diverse cellular targets. Biochem J. 2009;418:475-89.

56. Jeffrey KL, Brummer T, Rolph MS, Liu SM, Callejas NA, Grumont RJ, Gillieron C, Mackay F, Grey S, Camps M, et al. Positive regulation of immune cell function and inflammatory responses by phosphatase PAC-1. Nat Immunol. 2006;7:274-83.

57. Nguyen T, Nioi P, Pickett CB. The Nrf2-antioxidant response element signaling pathway and its activation by oxidative stress. J Biol Chem. 2009; 284:13291-5.

58. Martin-de-Saavedra MD, Budni J, Cunha MP, Gomez-Rangel V, Lorrio S, Del Barrio L, Lastres-Becker I, Parada E, Tordera RM, Rodrigues AL, et al. Nrf2 participates in depressive disorders through an anti-inflammatory mechanism. Psychoneuroendocrinology. 2013;38:2010-22.

59. Martin-Hernandez D, Bris AG, MacDowell KS, Garcia-Bueno B, Madrigal JL, Leza JC, Caso JR. Modulation of the antioxidant nuclear factor (erythroid 2-derived)like 2 pathway by antidepressants in rats. Neuropharmacology. 2016;103:79-91.

60. Bouvier E, Brouillard F, Molet J, Claverie D, Cabungcal JH, Cresto N, Doligez N, Rivat C, Do KQ, Bernard C, et al. Nrf2-dependent persistent oxidative stress results in stress-induced vulnerability to depression. Mol Psychiatry. 2017:22:1701-13.

61. Maes M, Fisar Z, Medina M, Scapagnini G, Nowak G, Berk M. New drug targets in depression: inflammatory, cell-mediated immune, oxidative and nitrosative stress, mitochondrial, antioxidant, and neuroprogressive pathways. And new 
drug candidates--Nrf2 activators and GSK-3 inhibitors. Inflammopharmacology. 2012;20:127-50.

62. Leonard B, Maes M. Mechanistic explanations how cell-mediated immune activation, inflammation and oxidative and nitrosative stress pathways and their sequels and concomitants play a role in the pathophysiology of unipolar depression. Neurosci Biobehav Rev. 2012;36:764-85.

63. Schmidt AJ, Heiser P, Hemmeter UM, Krieg JC, Vedder H. Effects of antidepressants on mRNA levels of antioxidant enzymes in human monocytic U-937 cells. Prog Neuro-Psychopharmacol Biol Psychiatry. 2008;32:1567-73.

64. Swisher JF, Burton N, Bacot SM, Vogel SN, Feldman GM. Annexin A2 tetramer activates human and murine macrophages through TLR4. Blood. 2010;115: 549-58.

65. Warner-Schmidt JL, Vanover KE, Chen EY, Marshall JJ, Greengard P. Antidepressant effects of selective serotonin reuptake inhibitors (SSRIs) are attenuated by antiinflammatory drugs in mice and humans. Proc Natl Acad Sci U S A. 2011;108:9262-7.

66. Svenningsson P, Chergui K, Rachleff I, Flajolet M, Zhang X, El Yacoubi M, Vaugeois JM, Nomikos GG, Greengard P. Alterations in 5-HT1B receptor function by p11 in depression-like states. Science. 2006;311:77-80.

67. Svenningsson P, Kim Y, Warner-Schmidt J, Oh YS, Greengard P. p11 and its role in depression and therapeutic responses to antidepressants. Nat Rev Neurosci. 2013;14:673-80.

68. Kaltschmidt B, Kaltschmidt C. NF-KappaB in long-term memory and structural plasticity in the adult mammalian brain. Front Mol Neurosci. 2015;8:69.

69. Steiner J, Bielau H, Brisch R, Danos P, Ullrich O, Mawrin C, Bernstein HG, Bogerts B. Immunological aspects in the neurobiology of suicide: elevated microglial density in schizophrenia and depression is associated with suicide. J Psychiatr Res. 2008;42:151-7.

70. Hawton K, van Heeringen K. Suicide. Lancet. 2009;373:1372-80.

71. Deep-Soboslay A, Akil M, Martin CE, Bigelow LB, Herman MM, Hyde TM, Kleinman JE. Reliability of psychiatric diagnosis in postmortem research. Biol Psychiatry. 2005;57:96-101.

Ready to submit your research? Choose BMC and benefit from:

- fast, convenient online submission

- thorough peer review by experienced researchers in your field

- rapid publication on acceptance

- support for research data, including large and complex data types

- gold Open Access which fosters wider collaboration and increased citations

- maximum visibility for your research: over $100 \mathrm{M}$ website views per year

At $\mathrm{BMC}$, research is always in progress.

Learn more biomedcentral.com/submissions 